INRA Prod. Anim.

1996, 9 (2), 133-139

\section{J.-B. COULON, F. DAUVER, J.-P. GAREL *}

\section{INRA Laboratoire Adaptation des Herbivores aux Milieux}

Theix 63122 Saint-Genès Champanelle

* INRA Domaine expérimental de La Borie

15190 Marcenat

\title{
Facteurs de variation de la numération cellulaire du lait chez des vaches laitières indemnes de mammites cliniques
}

La numération cellulaire du lait de troupeau constitue un élément important du paiement du lait. A l'échelle de l'animal ou du lait de tank, c'est un indicateur précieux de l'état sanitaire du troupeau (Poutrel 1985, Serieys 1992). Les cellules du lait sont en effet essentiellement constituées de globules blancs (lymphocytes, macrophages et leucocytes polynucléaires) provenant de la circulation sanguine et dont le nombre augmente considérablement en cas d'infection de la mamelle. Compte tenu de l'importance des mammites dans les troupeaux laitiers et de leurs conséquences sur les performances de production des animaux (Bartlett et al 1991, Lescourret et Coulon 1994), la qualité du lait (Munro et al 1984, Barbano et al 1991) et le revenu de l'éleveur (Beck et al 1992), il est important de bien connaître l'ensemble des facteurs de variation de la numération cellulaire du lait.

En dehors de l'état sanitaire de la mamelle, on observe en effet des variations parfois non négligeables de cette numération cellulaire.

\section{Résumé}

Les variations de la numération cellulaire du lait ont été analysées sur 404 lactations de 208 vaches laitières conduites sur un domaine expérimental de l'INRA et indemnes de mammites cliniques au cours des lactations considérées. En moyenne, la numération cellulaire a été minimale en $2^{\mathrm{e}}$ mois de lactation $(50000$ cellules/ml) et maximale en fin de lactation $(200000$ cellules $/ \mathrm{ml})$. Les vaches primipares ont présenté des numérations plus élevées que les multipares en début de lactation et plus faibles en fin de lactation. Les vaches Holstein ont présenté des numérations cellulaires constamment supérieures à celles des vaches Montbéliardes et Tarentaises. En fin de lactation, l'écart entre ces 2 groupes d'animaux atteint environ 120000 cellules $/ \mathrm{ml}$. Cet effet de la race n'est pas dû à un effet du niveau de production. Chez les vaches multipares, la présence de plus d'une mammite au cours des lactations précédentes a conduit à des numérations cellulaires significativement plus élevées (d'environ 40000 cellules/ml à l'échelle de la lactation) que chez les vaches ayant présenté une mammite ou pas du tout au cours des lactations précédentes. La numération cellulaire augmente légèrement, indépendamment de l'effet du stade de lactation, au cours des mois d'août et septembre. L'ensemble de ces facteurs de variations ne permettent cependant pas d'expliquer des numérations cellulaires supérieures à 300000 cellules/ml dans un lait de mélange.
Elle semble en particulier varier au cours de la lactation (Sheldrake et al 1983, Schutz et al 1990, Auldist et al 1995), et augmenter avec l'âge (Kennedy et al 1982, Schultz et al 1990), même chez des vaches indemnes de mammites (Serieys 1985a, Harmon 1994). Elle augmente aussi parfois en situation de stress (Wegner et al 1976) ou d'effort physique (J.B. Coulon et P. Pradel, non publié). Des variations saisonnières, parfois contradictoires, ont aussi été rapportées (Bodoh et al 1976, Kennedy et al 1982, Coulon et al 1988, Coulon et Lilas 1988). Cependant, dans la plupart de ces études, généralement réalisées à partir de données mensuelles du Contrôle Laitier, les vaches atteintes de mammites n'étaient pas exclues de l'analyse et les facteurs de conduite des vaches ainsi que leur passé sanitaire n'étaient pas toujours connus.

L'objectif de cette étude est de préciser, à partir de données recueillies de manière fréquente (toutes les 2 semaines) et issues de troupeaux expérimentaux conduits dans des conditions contrôlées et connues, les variations d'ordre physiologique de la numération cellulaire du lait chez des vaches indemnes de mammites cliniques.

\section{1 / Conduite de l'étude}

\section{1 / Origine des données}

Les données bimensuelles de numérations cellulaires individuelles (mesurées sur un échantillon de la traite du soir, à l'aide d'un appareil Somatocount 500) recueillies entre 1989 et 1994 sur le domaine expérimental de l'INRA à Marcenat (Cantal) ont été utilisées. Parmi les 642 lactations dont les données étaient disponibles sur cette période, seules ont été retenues celles au cours desquelles aucune mammite clinique (diagnostiquée lors de la traite par l'inflammation de la mamelle et la présence de grumeaux dans le lait) n'a été relevée et dont la durée était au moins 
égale à 33 semaines. L'étude a en définitive porté sur les laits produits au cours de 404 lactations de 208 vaches, Holstein $(n=147)$, Montbéliardes $(n=177)$ et Tarentaises $(n=80)$. Cet effectif inclut donc des lactations ayant pu présenter des numérations cellulaires élevées, du moment qu'elles n'ont pas été associées à des signes cliniques. Parmi les 208 vaches de cette étude, 41 ont été indemnes de mammites cliniques au cours de leurs 3 premières lactations (9 Holstein, 20 Montbéliardes et 12 Tarentaises).

Les vaches ont été conduites en stabulation entravée de début novembre à début mai (date moyenne de mise à l'herbe : 6 mai). elles participaient pour la plupart à des expérimentations concernant l'effet du niveau des apports alimentaires hivernaux sur les performances de production. Elles recevaient alors une ration composée d'ensilage d'herbe et/ou de foin offerte à volonté et complémentée par un aliment concentré à différents niveaux selon les protocoles expérimentaux. En été, les animaux étaient conduits ensemble sur des parcelles proches de l'étable où ils étaient ramenés 2 fois par jour pour la traite. Eté comme hiver, celle-ci avait lieu deux fois par jour (6 h et $15 \mathrm{~h} \mathrm{30),}$ en salle de traite. La majorité des vaches ont été inséminées artificiellement, sur chaleurs naturelles, entre 45 et 200 jours après le vêlage (tableau 1). Les vaches étaient taries 2 mois avant le vêlage suivant. Le tarissement était brutal et systématiquement accompagné d'un traitement antibiotique par voie intra mammaire.

Tableau 1. Répartition des 404 lactations étudiées selon leurs caractéristiques.

\begin{tabular}{|l|c|c|c|c|}
\hline Race & Tarentaise & Montbéliarde & Holstein & Total \\
\hline Numéro de lactation & & & & \\
1 & 23 & 60 & 60 & 143 \\
2 & 22 & 46 & 29 & 97 \\
3 & 16 & 32 & 29 & 77 \\
4 et 5 & 19 & 39 & 29 & 87 \\
\hline Mois de vêlage & & & & \\
Novembre ${ }^{(1)}$ & 38 & 64 & 52 & 154 \\
Décembre & 29 & 62 & 41 & 132 \\
Janvier-février & 12 & 48 & 44 & 104 \\
Mars-avril & 1 & 3 & 10 & 14 \\
\hline Stade de lactation au moment & & & & \\
de l'insémination fécondante & & & & \\
$<60$ j & 13 & 30 & 25 & 68 \\
60 -139 j & 62 & 125 & 98 & 285 \\
$>139$ j (2) & 5 & 22 & 24 & 51 \\
\hline & & & & Moyenne \\
Production laitière initiale (kg/j) & 14,6 & 17,0 & 22,5 & 18,6 \\
Production laitière totale $(\mathrm{kg})$ & 3128 & 4527 & 5594 & 4648 \\
Durée de lactation (semaines) & 38,4 & 39,5 & 39,2 & 39,2 \\
\hline
\end{tabular}

(1) Y compris quelques vêlages fin octobre.

(2) Y compris les vaches vides.
Figure 1. Répartition des 7930 numérations cellulaires réalisées sur les 404 lactations indemnes de mammites cliniques.

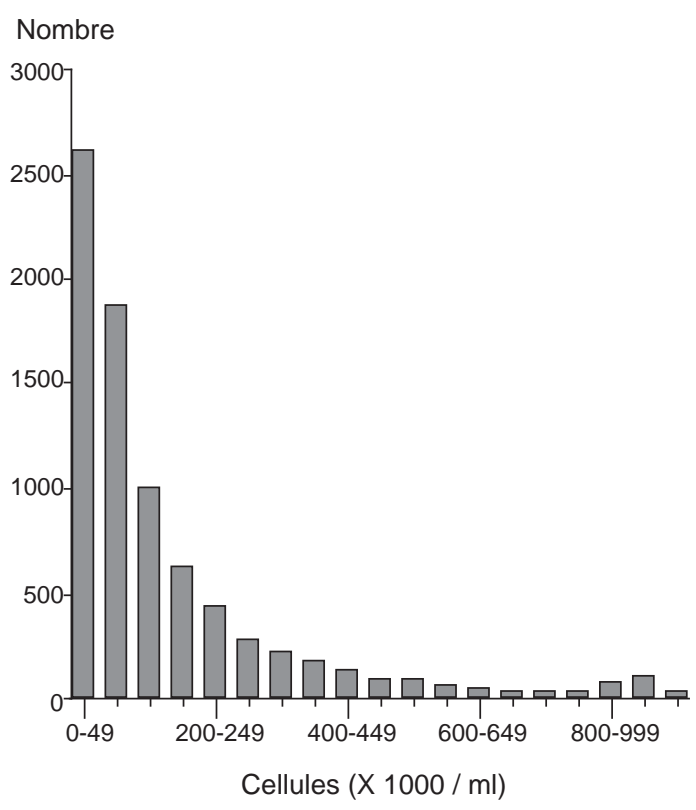

\section{2 / Analyse des données}

Pour chaque individu, les données ont été regroupées par quinzaine de jours après le vêlage (par exemple, une mesure réalisée entre le $15^{\mathrm{e}}$ et le $19^{\mathrm{e}}$ jour de lactation a été affectée à la quinzaine 2). Compte tenu de la répartition non normale des données (figure 1) l'évolution de la numération cellulaire du lait au cours de la lactation, pour l'ensemble des animaux ou pour des groupes d'animaux particuliers, a été réalisée en calculant pour chaque quinzaine, la moyenne géométrique des valeurs retenues.

Pour analyser l'effet des facteurs de variation, on a retenu 4 périodes particulières correspondant aux 2 premières quinzaine de lactation, aux quinzaines 6 à 8,12 à 14 et 18 à 20. Pour cette dernière période, seules 382 lactations ont été renseignées. On a par ailleurs calculé la moyenne géométrique des 17 premières quinzaines pour chaque individu. Après transformation logarithmique, ces 5 variables ont été traitées par analyse de variance en introduisant dans le modèle le numéro de lactation $(1,2,3$ et supérieur à 3 ), la race, le potentiel de production (estimé par la production initiale, moyenne des $4^{\mathrm{e}}$, $5^{\text {e }}$ et $6^{\text {e }}$ jours de lactation), la période de vêlage (regroupée en 4 classes : novembre, décembre, janvier-février et mars-avril), le stade de lactation au moment de l'insémination fécondante (regroupé en 3 classes : $<60$ j, 60-139 j, > 139 j), ainsi que, pour les lactations de rang 2 et plus, le nombre de mammites observées au cours des lactations précédentes (regroupé en 3 classes : 0, 1, 2 et plus). Les interactions entre les différents facteurs et la production initiale ainsi qu'entre eux n'ayant jamais été significatives, elles n'ont pas été retenues dans les analyses finales. 
Par ailleurs, l'effet du numéro de lactation a aussi été analysé en comparant les numérations cellulaires des 3 lactations des 41 vaches n'ayant jamais présenté de mammites cliniques au cours de leurs 3 premières lactations.

\section{2 / Résultats}

Sur l'ensemble des données disponibles $(\mathrm{n}=7930)$, la numération cellulaire a varié de 4000 à 7600000 cellules/ml (figure 1). Quatorze pour cent des valeurs observées ont été supérieures à 300000 et $1,7 \%$ ont été supérieures à 1000000 cellules $/ \mathrm{ml}$. Les valeurs ont été très faibles dans $8,1 \%$ des cas, inférieures ou égales à 20000 cellules/ml. La moyenne géométrique par lactation a varié de 21000 à 803000 cellules/ml. Pour $63 \%$ des lactations, la moyenne géométrique a été inférieure à 100000 cellules $/ \mathrm{ml}$ et pour $3,7 \%$ supérieure à 300000 cellules/ml.

\section{1 / Effet des facteurs physiologiques}

En moyenne, la numération cellulaire a évolué au cours de la lactation de façon inverse à la production laitière (figure 2). Elle a été minimale au cours du deuxième mois de lactation et maximale en fin de lactation. L'écart entre ces 2 périodes est, en moyenne, d'environ 150000 cellules/ml. L'augmentation est faible entre le $2^{\mathrm{e}}$ et le $7^{\mathrm{e}}$ mois (+ 30000 cellules $/ \mathrm{ml})$, mais plus importante entre le $7^{\mathrm{e}}$ et le $10^{\mathrm{e}}$ mois $(+120000$ cellules/ $\mathrm{ml})$. La proportion de prélèvements supérieurs à 300000 cellules $/ \mathrm{ml}$ a varié de $5 \%$ en deuxième mois de lactation, à $30 \%$ en $10^{\mathrm{e}}$ mois de lactation. Cette évolution a cependant présenté des variations sensibles selon les caractéristiques des vaches (tableau 2). En particulier, les vaches primipares ont présenté des numérations plus élevées que les multipares $(\mathrm{P}<0,01)$ en début de lactation, et plus faibles en fin de lactation $(\mathrm{P}<0,01)$. Si l'on calcule les quantités de cellules excrétées dans le lait, on observe que, en dehors du premier mois de lactation, celles-ci sont assez stables au cours de la lactation chez les primipares, mais doublent presque entre le $2^{\mathrm{e}}$ et le dernier mois de lactation chez les multipares (figure 3). Par ailleurs, les vaches Holstein ont présenté des numérations cellulaires constamment supérieures à celles des vaches des autres races $(\mathrm{P}<0,01)$; l'écart a été maximal en fin de lactation. Ces différences (entre races et entre numéros de lactation) ne sont pas liées aux variations du niveau de production des animaux : elles se maintiennent lorsque l'on compare des animaux de potentiel voisin, mais de race ou d'âge différents. Intra race et intra numéro de lactation, la numération cellulaire du lait a tendance à diminuer quand le potentiel de production augmente $(\mathrm{P}<0,1)$. Cet effet est cependant faible : en moyenne et à l'échelle de la lactation, la numération cellulaire dimi-
Figure 2. Evolution de la numération cellulaire (moyenne géométrique et fréquence des prélèvements supérieurs à 300000 cellules $/ \mathrm{ml}$ ) et de la production laitière au cours de la lactation chez les 404 lactations indemnes de mammites cliniques.

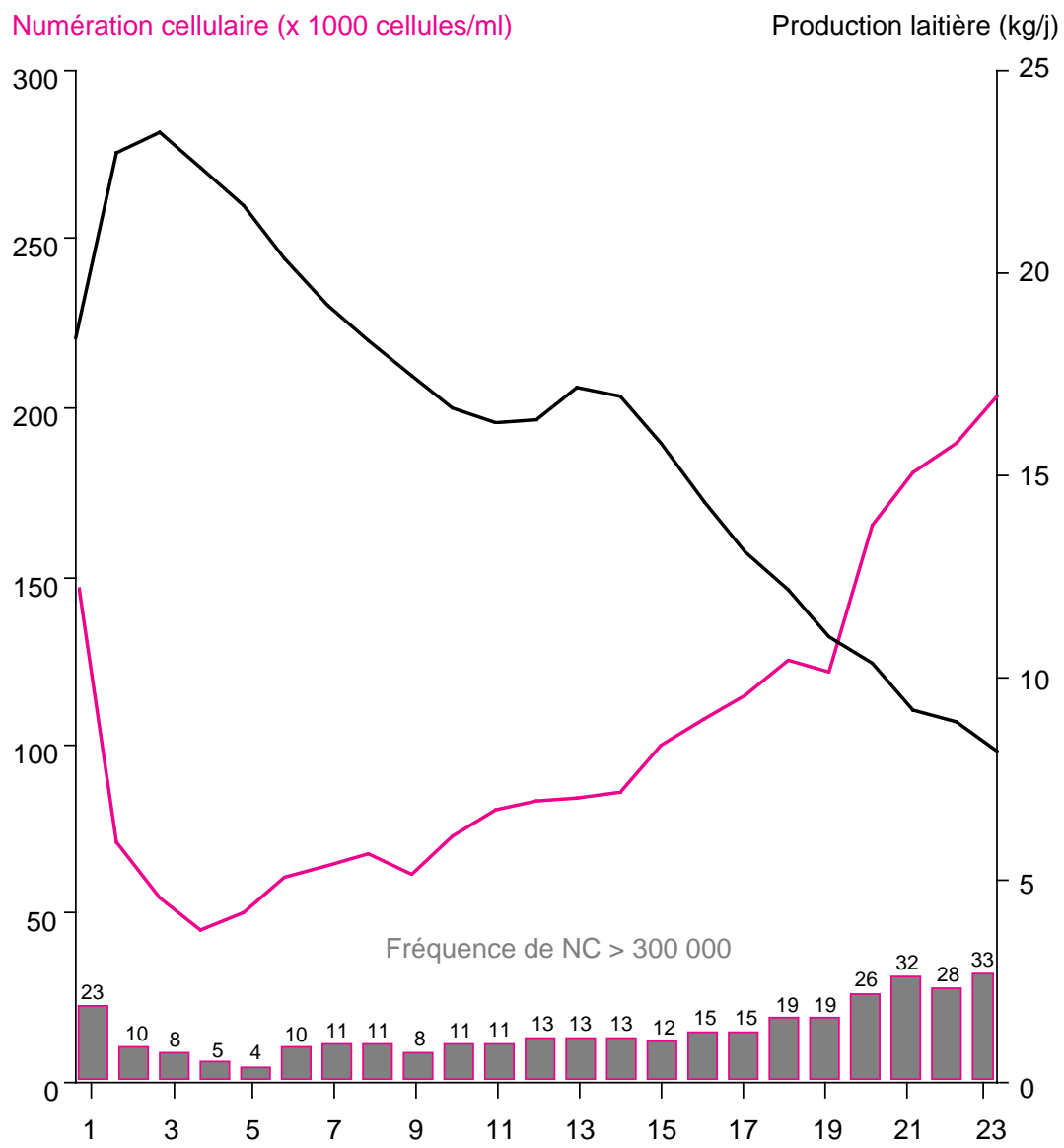

Tableau 2. Effet de la race, du numéro de lactation, de l'année, de la période de vêlage et, pour les 261 vaches multipares, de la présence de mammites au cours des lactations précédentes, sur la numération cellulaire du lait. Pour un facteur donné, à l'intérieur d'une colonne, les valeurs suivies d'une lettre différente sont significativement différentes $(P<0,01)$.

\begin{tabular}{|l|r|r|r|r|r|}
\hline \multirow{2}{*}{} & \multicolumn{4}{|c|}{ Quinzaines } \\
\cline { 2 - 5 } & $1-2$ & $6-8$ & $12-14$ & $18-20$ & $1-17$ \\
\hline \multirow{2}{*}{ Numéro de lactation } & & & & & \\
1 & $138 \mathrm{a}$ & 84 & $102 \mathrm{~b}$ & $118 \mathrm{a}$ & $83 \mathrm{ab}$ \\
2 & $70 \mathrm{~b}$ & 75 & $93 \mathrm{~b}$ & $157 \mathrm{~b}$ & $75 \mathrm{~b}$ \\
3 & $56 \mathrm{~b}$ & 81 & $111 \mathrm{ab}$ & $210 \mathrm{c}$ & $81 \mathrm{~b}$ \\
4 et 5 & $98 \mathrm{a}$ & 96 & $143 \mathrm{a}$ & $244 \mathrm{c}$ & $101 \mathrm{a}$ \\
\hline Race & & & & & \\
Holstein & $114 \mathrm{a}$ & $118 \mathrm{a}$ & $151 \mathrm{a}$ & $265 \mathrm{a}$ & $116 \mathrm{a}$ \\
Montbéliarde & $73 \mathrm{~b}$ & $64 \mathrm{~b}$ & $87 \mathrm{~b}$ & $137 \mathrm{~b}$ & $68 \mathrm{~b}$ \\
Tarentaise & $74 \mathrm{~b}$ & $78 \mathrm{~b}$ & $104 \mathrm{~b}$ & $148 \mathrm{~b}$ & $77 \mathrm{~b}$ \\
\hline Période de vêlage & & & & & \\
Novembre & $111 \mathrm{a}$ & 87 & $98 \mathrm{a}$ & 166 & 80 \\
Décembre & $86 \mathrm{~b}$ & 83 & $105 \mathrm{a}$ & 181 & 83 \\
Janvier-février & $76 \mathrm{~b}$ & 83 & $133 \mathrm{~b}$ & 192 & 88 \\
Mars-avril & $72 \mathrm{ab}$ & 81 & $109 \mathrm{ab}$ & 165 & 87 \\
\hline Année & & & & & \\
1988-89/89-90/90-91/92-93 & $79 \mathrm{a}$ & $77 \mathrm{ab}$ & $118 \mathrm{a}$ & $180 \mathrm{ab}$ & $79 \mathrm{a}$ \\
1991-92 & $126 \mathrm{~b}$ & $103 \mathrm{~b}$ & $130 \mathrm{a}$ & $203 \mathrm{a}$ & $108 \mathrm{~b}$ \\
$1993-94$ & $62 \mathrm{a}$ & $74 \mathrm{a}$ & $88 \mathrm{~b}$ & $148 \mathrm{~b}$ & $70 \mathrm{a}$ \\
\hline Nombre de mammites au cours des lactations précédentes & & \\
0 & $57 \mathrm{a}$ & $67 \mathrm{a}$ & $87 \mathrm{a}$ & $157 \mathrm{a}$ & $69 \mathrm{a}$ \\
1 & $64 \mathrm{a}$ & $75 \mathrm{ab}$ & $105 \mathrm{a}$ & $167 \mathrm{ab}$ & $77 \mathrm{a}$ \\
2 et + & $111 \mathrm{~b}$ & $108 \mathrm{~b}$ & $167 \mathrm{~b}$ & $214 \mathrm{~b}$ & $116 \mathrm{~b}$ \\
\hline
\end{tabular}


Figure 3. Evolution du nombre de cellules excrétées au cours de la lactation selon son rang.

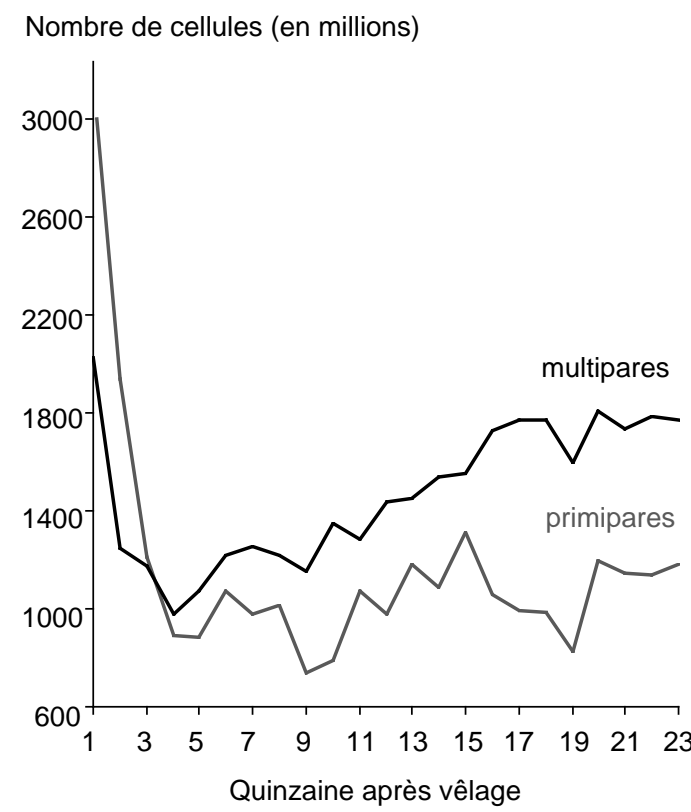

nue d'environ 10000 cellules/ml quand la production initiale augmente de $10 \mathrm{~kg} / \mathrm{j}$.

La numération cellulaire n'a pas dépendu du stade de gestation : en fin de lactation, la numération cellulaire n'a pas été significativement différente selon que les vaches étaient gestantes depuis plus de 200 jours (168 000 cellules/ml) ou depuis moins de 120 jours (191000 cellules/ml).

Chez les vaches multipares, la présence de plus d'une mammite au cours des lactations précédentes a conduit à des numérations cellulaires significativement plus élevées (d'environ 40000 cellules $/ \mathrm{ml}$ à l'échelle de la lactation) que chez les vaches ayant présenté une mammite clinique ou pas du tout au cours des lactations précédentes.

\section{2 / Effet de la saison et de l'année}

La période de vêlage a eu un effet significatif sur la numération cellulaire en début (quinzaines 1 et 2 ) et en milieu de lactation (quinzaines 12 à 14). Les numérations cellulaires ont été supérieures d'environ 30000 cellules/ml en début de lactation chez les vaches vêlant en novembre, c'est-à-dire en tout début de période de stabulation hivernale. Les numérations cellulaires ont aussi été supérieures $(\mathrm{P}<0,01)$ en milieu de lactation chez les vaches ayant vêlé en janvierfévrier, comparativement à celles ayant vêlé en novembre. Il existe ainsi une interaction significative $(\mathrm{P}<0,01)$ entre le stade de lactation et la période de vêlage. Ce résultat peut être interprété comme un effet propre de la saison, indépendamment des autres facteurs introduits dans le modèle. Ainsi, chez toutes les vaches, quelle que soit la période de vêlage, on observe une augmentation de la numération cellulaire au cours des mois d'août et septembre. Corrigé pour tenir compte du stade de lactation, cet effet est de l'ordre de 80000 cellules $/ \mathrm{ml}$, entre mai et septembre (figure 4). L'évolution conjointe de la température moyenne observée est relativement faible $\left(+5^{\circ} \mathrm{C}\right.$ entre mai et septembre) et il est peu probable qu'elle soit la cause de l'augmentation de la numération cellulaire. La mise à l'herbe (réalisée au début du mois de mai) ne s'est pas accompagnée de modifications sensibles de la numération cellulaire du lait.

En moyenne, les numérations cellulaires ont été significativement supérieures (d'environ 30000 cellules $/ \mathrm{ml}$ ) au cours de la campagne 91/92 comparativement aux autres. Parmi celles-ci, la campagne 93/94 a été caractérisée par des numérations cellulaires faibles, en particulier en milieu de lactation.

\section{3 / Discussion}

L'absence d'indicateur objectif de l'état sanitaire de la mamelle (présence et nature des principaux germes responsables de mammites) et de caractéristiques plus fines de la nature des cellules du lait, confère à cette étude un caractère essentiellement descriptif. Nous n'avons ainsi pas pu tenir compte des infections subcliniques qui représentent, en l'absence de mammite clinique, un facteur majeur de variation de la numération cellulaire du lait. Son originalité réside dans le fait qu'elle porte sur des lactations indemnes de mammites cliniques, conduites dans des conditions de milieu les plus comparables possibles, y compris dans le temps. L'effet de l'année, que l'on pourrait associer à celui des variations des conditions de milieu (hygiène, conduite de la traite...) au cours du temps

Figure 4. Evolution de la numération cellulaire du lait en fonction de la saison, à stade de lactation comparable (6-7e mois).

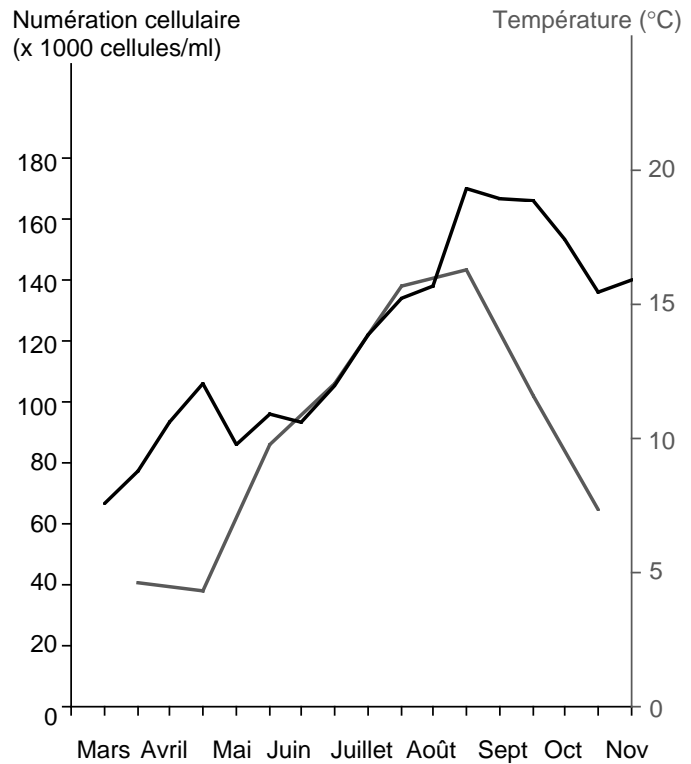


apparaît ainsi relativement limité, en dehors de la campagne 91-92. Une hypothèse d'explication de cet effet année est que certaines vaches réagiraient à un environnement microbien défavorable (responsable d'une fréquence élevée de mammites cliniques dans le troupeau) par une augmentation de leur numération cellulaire sans apparition de mammite clinique. Il est cependant intéressant de noter que si la campagne 91-92 a été caractérisée par une fréquence élevée de mammites cliniques, elle n'est pas la seule (la campagne 90-91 présente la même fréquence). C'est par contre la campagne où les mammites estivales ont été les plus fréquentes (32 mammites observées sur le troupeau entre le 10 mai et le $1^{\text {er }}$ octobre, contre 10 à 15 les autres années).

Cette étude a montré que, même chez des vaches indemnes de mammites cliniques, la numération cellulaire du lait pouvait présenter des variations sensibles. La fréquence de valeurs très élevées ( $>1$ million de cellules $/ \mathrm{ml})$ est cependant faible $(1,7 \%)$, du même ordre de grandeur que celle observée par Serieys (1985b) chez des vaches non infectées par des germes pathogènes majeurs ou mineurs $(1,2 \%)$. Même si cela reste possible, il est peu probable, dans les conditions de recueil des données de cette étude, que ces valeurs soient dues à des mammites cliniques non relevées (et donc non soignées). La plupart de ces valeurs apparaissent isolées au cours d'une lactation (les 136 valeurs supérieures à 1 million de cellules/ml concernent 84 lactations et 70 vaches différentes). Il est possible, bien que nous n'ayons pas pu le vérifier, que ces valeurs témoignent d'un stress de l'animal ou d'une modification de son statut physiologique (œstrus), associé ou non à une diminution sensible de la production, ayant pu accentuer, par effet de concentration, l'augmentation de la numération cellulaire. Il est aussi possible que certaines vaches réagissent fortement à une infection sans développer de signes cliniques (ce qui correspond à la définition d'une mammite subclinique). Cependant, il faut noter que les numérations cellulaires élevées observées de manière isolée en milieu de lactation n'ont pas été associées à une chute de production laitière, contrairement à ce que l'on observe le plus souvent dans le cas de mammite clinique (Lescourret et Coulon 1994).

Comme cela a été montré par de nombreux auteurs (Wood et Booth 1983, Ng-Kwai-Hang et al 1984, Serieys 1985a, Schutz et al 1990, Auldist et al 1995), deux facteurs importants des variations de la numération cellulaire sont le stade et le numéro de lactation. Notre étude montre que l'augmentation de la numération cellulaire au cours de la lactation ne peut pas être imputée à des mammites cliniques ayant eu lieu en début ou milieu de lactation. Cette augmentation est plutôt due à un effet de concentration (Kennedy et al 1982, Sheldrake et al 1983), au moins chez les primipares où la quantité de cellules excrétées reste pratiquement stable au cours de la lac- tation. Ce n'est pas le cas chez les multipares, peut-être en raison d'une diminution de la quantité de cellules sécrétrices plus importante que chez les primipares. Il est donc possible qu'en fin de lactation les cellules épithéliales participent à l'augmentation de la numération cellulaire (Blackburn 1966), en particulier chez les vaches multipares, bien que cette hypothèse ne soit pas toujours admise (Harmon 1994). En début de lactation, chez les primipares, le niveau élevé de la numération cellulaire que nous avons observé confirme les observations antérieures (Schutz et al 1990). Son origine serait plus physiologique (mise en place de la lactation, faible production laitière), ou lié à des œdèmes, qu'infectieuse (Schutz et al 1990). Notre étude permet de confirmer indirectement cette origine physiologique : c'est à cette période et chez les vaches primipares que l'effet du niveau de production est apparu le plus important $(\mathrm{P}<0,01)$. Ce résultat va dans le sens des observations de Philipot (1995) qui a mis en évidence, en début de lactation, une relation inverse entre le niveau de production laitière et la numération cellulaire de vaches non infectées par des germes pathogènes. L'effet de l'âge que nous avons observé est certainement dû, au moins en partie (Serieys 1985a), à la présence d'infections mammaires au cours des lactations précédentes. Notre étude montre qu'il faut que ces infections soient multiples pour avoir un effet au cours d'une lactation ultérieure indemne de mammite clinique. Elle montre aussi, contrairement à certains travaux (Sheldrake et al 1983, Badinand 1994), qu'une partie de l'effet de l'âge pourrait être d'origine physiologique

Figure 5. Effet du numéro de lactation sur la numération cellulaire du lait, chez les 41 vaches indemnes de mammites cliniques au cours de leurs 3 premières lactations. (moyennes ajustées en tenant compte de la race, de la période de vêlage, du stade de gestation et de la campagne).

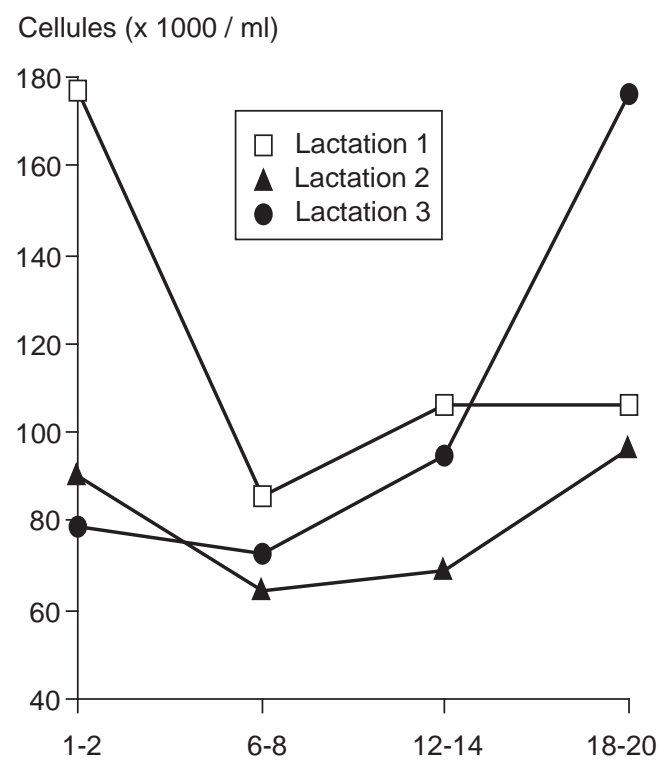

Quinzaines de lactation
La numération cellulaire est plus élevée chez les Holstein que chez les Montbéliardes et les Tarentaises. 
Figure 6. Evolution de la numération cellulaire du lait de tank et de la production laitière journalière dans 2 situations différentes :

a - troupeau âgé de 120 vaches Holstein (30\% de primipares, $18 \%$ de lactations de rang supérieur ou égal à 4) présentant des vêlages groupés entre début novembre et fin février.

b - troupeau jeune de 155 vaches Montbéliardes (38\% de primipares, $12 \%$ de lactations de rang supérieur ou égal à 4) présentant des vêlages répartis entre début novembre et mi-avril.
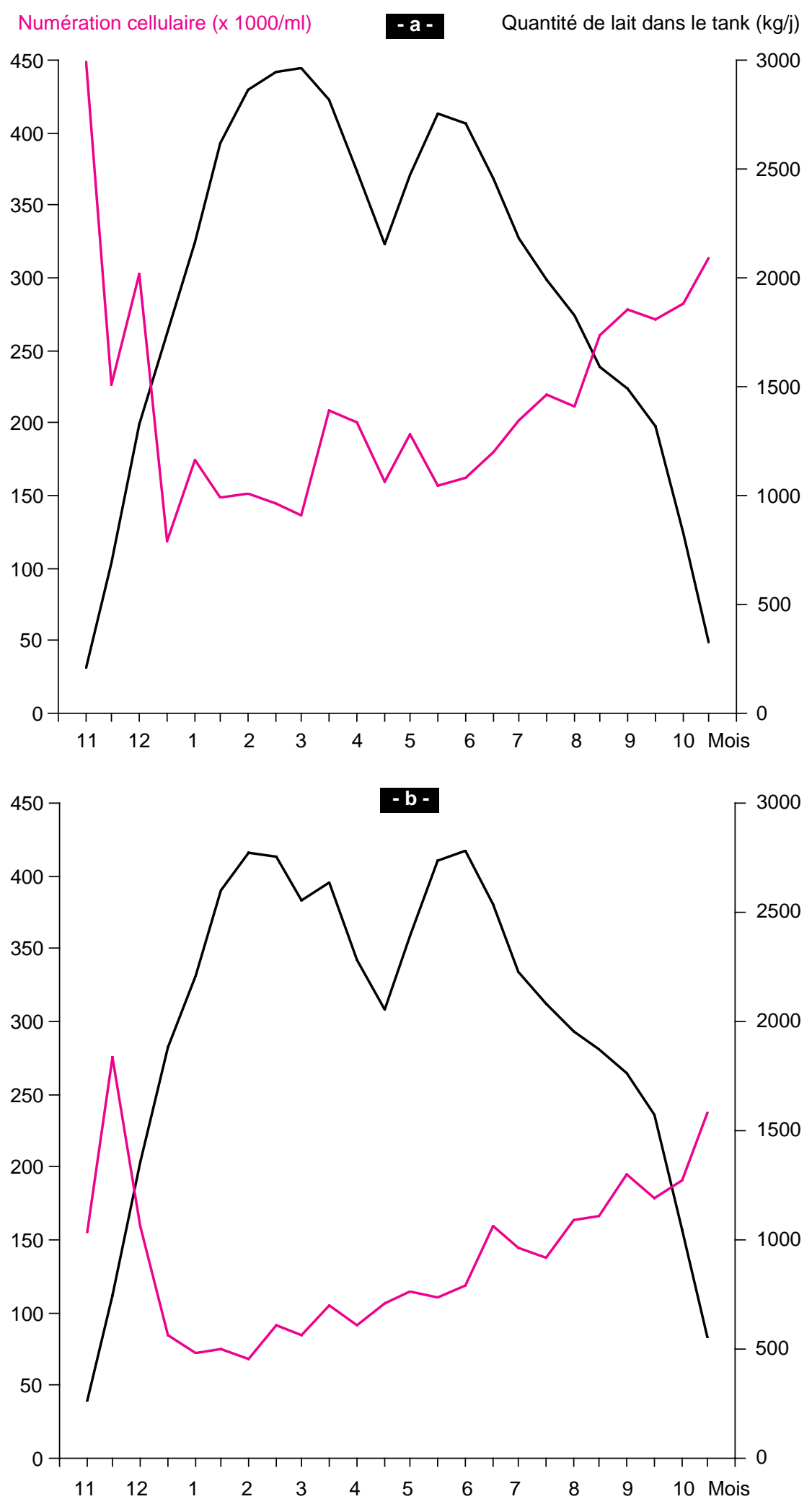

(Harmon 1994) : cet effet se maintient en effet de manière significative lorsque l'on compare les lactations successives de vaches $(n=41)$ n'ayant jamais présenté de mammites cliniques au cours de leurs 3 premières lactations (figure 5), ce qui confirme les observations de Serieys (1985a) chez des vaches non infectées par des germes pathogènes.

Cette étude a également mis en évidence un effet important de la race sur la numération cellulaire. Cet effet ne peut pas être confondu avec un effet de la présence de mammites cliniques au cours des lactations précédentes (plus fréquentes chez les vaches Holstein dans le troupeau étudié) : l'effet de la race se maintient de manière significative lorsque l'on ne considère que les vaches primipares ou que les vaches ayant présenté 3 lactations successives indemnes de mammites, ou que l'on tient compte, dans l'analyse réalisée sur les lactations de rang supérieur à 1 , de la présence de mammites cliniques au cours des lactations précédentes. Les raisons de cet effet de la race restent à préciser : ils peuvent être dus à une fréquence plus grande de mamelles infectées (sans apparition de mammite clinique) chez les vaches Holstein, en relation avec des caractéristiques physiques de trayons plus favorables à la pénétration des microbes (Jorstad et al 1989).

Ces facteurs physiologiques de variation de la numération cellulaire du lait concernant des lactations indemnes de mammites cliniques ne permettent d'expliquer, dans nos conditions d'observation, qu'entre 9 et $22 \%$ de la variabilité totale ( $15 \%$ pour la valeur moyenne à l'échelle de la lactation). Lorsque l'on cumule les effets du stade de lactation, de l'âge et de la race, on peut cependant observer des écarts sensibles : ainsi, des vaches primipares Montbéliardes présentent en fin de lactation des numérations cellulaires 3 fois inférieures à celles de vaches Holstein en lactation 3 et plus (respectivement 110000 et 295000 cellules $/ \mathrm{ml}$ en $9^{\mathrm{e}}$ mois de lactation). Cependant, même dans ce dernier cas, la valeur moyenne (géométrique) ne dépasse pas, dans les conditions de conduite de notre étude, 300000 cellules $/ \mathrm{ml}$. En pratique, l'effet des facteurs de variation physiologiques que nous avons considérés reste ainsi relativement limité. A partir des données de cette étude, on peut reconstituer un lait de tank provenant de vaches présentant des caractéristiques variables, mais toutes indemnes de mammites cliniques pour la lactation considérée. La comparaison de deux situations différentes (troupeau jeune de vaches Montbéliardes présentant des vêlages répartis entre début novembre et mi-avril, ou troupeau âgé de vaches Holstein présentant des vêlages groupés entre début novembre et fin février) montre que, dans les 2 cas, tant que le seuil $\mathrm{du}$ paiement du lait ne descend pas en dessous de 300000 cellules en moyenne, pratiquement aucun prélèvement ne sera soumis à pénalité (figure 6). 


\section{Références bibliographiques}

Auldist M.J., Coats S., Rogers G.L., McDowell G.H., 1995. Changes in the composition of milk from healthy and mastitic dairy cows during the lactation cycle. Austr. J. Exp. Agr., 35, 427-436.

Badinand F., 1994. Maîtrise du taux cellulaire du lait. Rec. Méd. Vét., 170, 419-427.

Barbano D.M., Rasmussen R.R., Lynch J.M., 1991. Influence of milk somatic cell count and milk age on cheese yield. J. Dairy Sci., 74, 369-388.

Bartlett P.C., Van Wijk J., Wilson D.J., Green C.D., Miller G.Y., Majewski G.A., Heider L.E., 1991. Temporal patterns of lost milk production following clinical mastitis in a large Michigan Holstein herd. J. Dairy Sci., 74, 1561-1572.

Beck H.S., Wise W.S., Dodd F.H., 1992. Cost-benefit analysis of bovine mastitis in the UK. J. Dairy Res., 59, 449-457.

Blackburn P.S., 1966. The variation in the cell count of cow's milk throughout lactation and from one lactation to the next. J. Dairy Res., 33, 193-198.

Bodoh G.W., Battista W.J., Schultz L.H., Johnston R.P., 1976. Variation in somatic cell counts in dairy herd improvement milk samples. J. Dairy Sci., 59, 1119-1123.

Coulon J.B., Lilas J.P., 1988. Composition chimique et contamination butyrique du lait : facteurs de variation dans le département de la Haute-Loire. INRA Prod. Anim., 1, 201-207.

Coulon J.B., Roybin D., Congy E., Garret A., 1998. Composition chimique et temps de coagulation du lait de vache : facteurs de variations dans les exploitations du Pays de Thônes. INRA Prod. Anim., 1, 253-263.

Harmon R.J., 1994. Physiology of mastitis and factors affecting somatic cell counts. J. Dairy Sci., 77, 2103-2112.

Jorstad A., Farver T.B., Riemann H., 1989. Teat canal diameter and other cow factors with possible influence on somatic cell counts in cow milk. Acta. Vet. Scand., 30, 239-245.

Kennedy B.W., Sethar M.S., Tong A.K.W.W., Moxley J.E., Downey B.R., 1982. Environmental factors influencing test-day somatic cell counts in Holsteins. J. Dairy Sci., 65, 275-280.
Lescourret F., Coulon J.B., 1994. Modeling the impact of mastitis on milk production by dairy cows. J. Dairy Sci., 77, 2289-2301.

Munro G.L., Grieve P.A., Kitchen B.J., 1984. Effects of mastitis on milk yield, milk composition, processing properties and yield and quality of milk products. Aust. J. Dairy Tech., 39, 7-16.

Ng-Kwai-Hang K.F., Hayes J.F., Moxley J.E., Monardes G., 1984. Variability of test-day milk production and composition and relation of somatic cell counts with yield and compositional changes of bovine milk. J. Dairy Sci., 67, 361-366.

Philipot J.M., 1995. Dynamique de la concentration cellulaire du lait de vache. Caractéristiques des infections mammaires dues aux principaux germes pathogènes. DEA, Université C. Bernard, Lyon I, 77 pp.

Poutrel B., 1985. Généralités sur les mammites de la vache laitière. Processus infectieux, épidémiologie, diagnostic, méthodes de contrôle. Rec. Méd. Vét., 161, 497-511.

Schutz M.M., Hansen L.B., Steuernagel G.R., Kuck A.L., 1990. Variation of milk, fat, protein, and somatic cells for dairy cattle. J. Dairy Sci., 73, 484-493.

Serieys F., 1985a. Concentration cellulaire du lait individuel de vache : influence de l'état d'infection mammaire, du numéro de lactation, du stade de lactation et de la production laitière. Ann. Rech. Vét., $16,255-261$.

Serieys F., 1985b. Interprétation des concentrations cellulaires du lait individuel de vache pour le diagnostic de l'état d'infection mammaire. Ann. Rech. Vét., 16, 263-269.

Serieys F., 1992. Les mammites des vaches laitières. Collection "Le point sur ", Institut de l'Elevage, Paris.

Sheldrake R.F., Hoare R.J.T., Mc Gregor G.D., 1983. Lactation stage, parity and infection affecting somatic cells, electrical conductivity and serum albumin in milk. J. Dairy Sci., 66, 542-547.

Wegner T.N., Schuh J.D., Nelson F.E., Stott G.H., 1976. Effect of stress on blood leucocyte and milk somatic cell counts in dairy cows. J. Dairy Sci., 59, 949-956.

Wood P.D.P., Booth J.M., 1983. Variation in milk cell counts during lactation of british Friesian cattle. Anim. Prod., 36, 335-339.

\section{Abstract}

Changes in somatic cell count in dairy cows free of clinical mastitis.

Changes in somatic cell count were analysed in 404 lactations of 208 dairy cows managed on an INRA experimental farm. Only lactations free of clinical mastitis were included in this study. On average, somatic cell count was minimal in the second month of lactation (50 000 cells $/ \mathrm{ml})$ and maximal in late lactation (200 000 cells $/ \mathrm{ml})$. Primiparous cows showed higher somatic cell count than multiparous cows in early lactation, and lower in late lactation. Somatic cell count was higher in Holstein cows than in Montbéliarde or Tarentaise cows. In late lactation, the difference between these two groups of breeds reached 120 000 cells $/ \mathrm{ml}$. This effect of breed is not linked to milk yield. Multiparous cows that were affected by more than one clinical mastitis during the preceeding lactations showed higher somatic cell count $(+40000$ cells $/ \mathrm{ml}$ over the whole lactation) than those that have had one or no clinical mastitis during the preceeding lactations. Somatic cell count increased slightly during summer, independently of lactation stage. However, all these physiological variation factors cannot explain bulk milk somatic cell count higher than 300000 cells $/ \mathrm{ml}$.

COULON J.B., DAUVER F., GAREL J.P., 1996. Facteurs de variation de la numération cellulaire du lait chez des vaches laitières indemnes de mammites cliniques. INRA Prod. Anim., 9 (2), 133-139. 